Informatics Engineering, an International Journal (IEIJ), Vol.4, No.4, December 2016

\title{
PRACTICE OF CALIBRATION TECHNOLOGY FOR THE SPECIAL TEST EQUIPMENT
}

\author{
Xu Yi-xiong ${ }^{1,2}$ Mu Xiao-nian ${ }^{2}$ \\ 1, Shanghai University of Engineering Science, Shanghai ,China \\ 2, Shanghai Institute of Spaceflight Control Technology, Shanghai ,China
}

\begin{abstract}
For the issues encountered in the special test equipment calibration work, based on the characteristics of special test equipment, the calibration point selection,classification of calibration parameters and calibration method of special test equipment are briefly introduced in this paper, at the same time, the preparation and management requirements of calibration specification are described.
\end{abstract}

\section{KEYWORD}

Special Test Equipment;Calibration parameter;Calibration Method;Calibration specification

\section{CharaCteristics OF SPECIAL TeST EQUiPMENT}

With the development of many models in our country, all kinds of special test equipment for the model are designed to provide testing support ${ }^{[1-2]}$.Test parameters involved in radio, electrical, microwave, optical, thermal, mechanical, length and other metrology specialties. Comprehensive induction of these devices, they have the following characteristics:

a) Multi-parameter test. It not only has a single parameter, as well as integrated parameter;

b) Measurement parameters are widely distributed,involving a total of eight major specialties;

c) High degree of automation.With the continuous development of computer technology and the popularization and application of test technology, in order to improve the efficiency of research and production testing, the majority of special test equipment are the automatic control system;

d) Huge volume of equipment.Most of the special test equipment are the integration of a variety of units, whose large size makes them not easy to transport;

e) Site measurement.Due to the equipment being not suitable to leave the field of research and production, calibration work have to be carried out in the field;

f) A wide range of test interfaces.Most special test equipment is not set specific calibration interface,so calibration works only with the aid of the test interface of the equipment;

Need to develop a dedicated calibration method. Because of the diversity of special test equipment, there is no ready-made, available, unified test procedures or calibration specifications.

DOI : 10.5121/ieij.2016.4401 
Informatics Engineering, an International Journal (IEIJ), Vol.4, No.4, December 2016

\section{SPECIAL TEST EQUIPMENT CALIBRATION}

\subsection{Selection of calibration points}

The vast majority of dedicated test equipment are connected to tested products by air plugs and proprietary cables. The cable is the main link between the special test equipment and the tested product, which is responsible for the transmission task of almost all electrical signals.General test equipment has a standard input / output terminals, while the special test equipment has no unified standard interface,so the main problem that we meets is the choice of the calibration position point.If the calibration points selected is not appropriate, it is possible to make the test data does not reflect the true state of equipment.Substandard equipment may be sentenced to be qualified, qualified equipment may be sentenced to be substandard.For example, when calibrating some equipment with load, if the calibration point is selected at the outlet end unit of the apparatus, the measured parameters can meet the technical requirements. If the calibration point is the output port of the cable,because of line drop,the measured parameters ,in contrast,can not meet the technical requirements.In another case,some equipment has been modified by the software to measure the data,qualified in the output port of the test cable, while unqualified at the outlet end unit of the apparatus. The cable is a unique transmission channel between the special test equipment and the tested product, which is an integral part of the special testing equipment. That whether the design of the cable is reasonable or not is directly affect the accuracy of the test data. The output port of the cable should be taken as the calibration point during calibrating of the special test equipment ${ }^{[3]}$.

\subsection{The classification of calibration parameters}

Special test equipment as a complete system, is not the superposition of simple equipment unit, but an organic whole.Some factors, such as connections within the system, characteristic impedance matching,the degree of electromagnetic compatibility, power supply system,surrounding environment,can affect the accuracy of measurement results.So under the condition of the best possible condition,adopting in-situ calibration is the closest the real working state of the system, and special test equipment technical indicators are usually lower than the corresponding general equipment.Therefore, with the high integration of the general test equipment, VXI module, PXI module setting up a standard device conditions, it can achieve a semi-automatic or fully automatic calibration work, and then achieve the traceability of special test equipment, standard device for special test equipment, metrological standards and national defense or national standard.The parameters which special test equipment need to calibrate are much more,such as single parameter and comprehensive parameter.The following are the calibration methods of these two parameters.

\subsubsection{Calibration of the single parameter}

For the single parameter calibration,the following takes a model product radio integrated calibration device as an example. In order to facilitate the development of a comprehensive calibration device, and according to a special test equipment used for a certain type of product, we analyze synthetically the characteristics of the cable transmission signal between the special test equipment and the tested products. According to its attribute, range, accuracy grade classification,the calibration parameters of a specific test equipment for a certain type of product specific test equipment are classified as follows: 
Informatics Engineering, an International Journal (IEIJ), Vol.4, No.4, December 2016

\section{a) DC signal}

The DC voltage signals are divided according to the input and output characteristics, which mainly comprises a standard voltage excitation class and an output voltage collection class. The standard excitation voltage and the output voltage are classified according to the range size and the accuracy grade.

\section{b) Loading signal}

Loading signal whose power usually is relatively large is mainly related to the power supply parameters. The specification, material and wire diameter of transmission line directly influence the quality of power supply.In order to facilitate calibration system design, the loading signal is classified in a centralized manner.

\section{c) Low frequency signal}

The signal lower than $100 \mathrm{kHz}$ is classified as low frequency.In order to avoid the interference of AC signal in the calibration system, the low frequency signal and the DC signal are separately processed.According to the frequency range, amplitude and accuracy of the low frequency signal,it can be transmitted by twisted pair in the calibration system.

\section{d) High frequency signal}

The signal is classified as high frequency signal in the range of $100 \mathrm{kHz} \sim 300 \mathrm{MHz}$. Because high frequency signal transmission and measurement requirements are relatively high, and the transmission line characteristics, impedance matching, attenuation and other factors all affect the measurement of high frequency signals, the high frequency signals are grouped together and divided according to the frequency, amplitude and the accuracy.High frequency signal transmission firstly ensure the signal source, transmission line, terminal in the best matching state.Based on the above mentioned parameters, the calibration interface of the integrated calibration device is designed,Calibration interface is designed into four types, which are the DC signal calibration interface, the loading signal calibration interface, the low frequency signal calibration interface and the high frequency signal calibration interface.The physical connection mode of calibration interface should be determined according to the character and quantity of the transmission signal.

\subsubsection{Calibration of comprehensive parameters}

Comprehensive parameters are related to a number of input variables, and to meet a certain functional relationship, which can be expressed as a comprehensive parameter $\mathrm{Y}$ is a function of $\mathrm{m}$ inputs-X1, $\mathrm{X} 2, \ldots \mathrm{Xm}, \mathrm{Y}=\mathrm{F}(\mathrm{X} 1, \mathrm{X} 2, \cdots, \mathrm{Xm})$. The $\mathrm{Xi}$ may be related to each other. The methods we often use for the calibration of the comprehensive parameters are as follows:

\section{a) Indirect calibration method}

Calibrating comprehensive parameters directly can not be achieved or more difficult.By indirectly measuring the input, and based on the known function of the relationship,we can drive the size of the comprehensive parameters, as is the method called the indirect calibration method. For 
example,we use rotary table to measure the IR seeker lock quality ${ }^{[4]}$. Since it is difficult to measure directly the electricity quality parameter $\eta$,we can indirectly measure it according to the formula (1):

$$
\eta=u_{1} u_{2} \cdot q_{g 0} \psi_{0}
$$

In the formula:

$u_{1} \ldots$ _ Off axis angle signal 1 of the seeker ;

$u_{2} \_$_ Off axis angle signal 2 of the seeker;

$q_{g 0} \ldots$ _ Angular velocity of the seeker;

$\psi_{0} \ldots$ _ Initial off axis angle of the seeker.

When using the method of indirect calibration, we should pay special attention to the approximate formula derived from the simplified formula, because the calculated value will have a large deviation.At this time, the calculated value can not be treated as a standard value, otherwise it will affect the judgment of the measurement equipment technical state.In this case, Agreed true value, standard sample verification or other processing method can be adopted.

\section{b) The calibration method of standard sample verification}

For some comprehensive parameters, it is difficult to establish the function relationship between the output and the input quantity.Sometimes, the function relation is an approximate expression, and due to the limitation of technical conditions, direct or indirect calibration can't be realized for the time being.Then we must use standard samples, using the sample repetition of standard value as the standard value of calibration equipment.For example,since the noise equivalent temperature difference of infrared imaging system(NETD), is related to the focal length of the optical system, viewing angle, frame, scanning efficiency, instantaneous field of view angle, pupil size, spectral transmittance, detector detection, spectral radiant exitance, target band parameters, which is very difficult to be measured directly or indirectly,to carry out calibration of measuring equipment must be with the help of standard samples - the product standard process parts.Standard sample is the Vector of value transfer.Using standard craft parts as standard samples,first, the process must be confirmed by strict examination.For example,after a rigorous field test,we confirm that the product meets the design requirements and get technical state stability;Second,like other measurement standards, the standard craft parts should be carried out in accordance with the provisions of the management and assessment.Because the technical state of standard samples is affected by device aging and environmental factors, how to ensure the state is the key.The daily assessment of standard samples should be achieved by periodic verification, statistical analysis, sampling inspection, technical evaluation method etc.

\section{CALIBRATION SPECIFICATION FOR THE SPECIAL TEST EQUIPMENT}

Calibration specification is the basis for calibration of the special test equipment.Because of the particularity of special test equipment, the corresponding calibration specification is to be prepared for its equipment.How to establish a scientific, reasonable and operable calibration 
specification is the premise to ensure the state of special test equipment.The following talks about the preparation of calibration specifications and management requirements.

\section{a) The preparation calibration specification is based on JJF1071-2010 - 《The Rules for Drafting National Specification of Calibration》.}

The compilation of specifications shall include measurement characteristics, calibration conditions, calibration items, calibration methods, and calibration records.In order to facilitate the unified management of calibration specifications,the competent authorities should design calibration specification template, and make detailed specification preparation requirements.

\section{b) Operability of calibration specifications}

That the calibration method is scientific and reasonable or not affects directly the calibration of equipment.Since designers are more familiar with the device itself,the preparation of calibration standards should be based on designers and equipment users.

\section{c) Examination of calibration specifications}

The examination of calibration specifications is in charge of the measurement supervisor.The main focus of the review is whether the specification is in compliance with JJF1071-2010, whether the measurement parameters can be traced back to the source, and whether calibration method steps are detailed and accurate, and so on.

\section{d) Validation of calibration specifications}

Calibration specification is the basis for acceptance and periodic calibration of the special test equipment.Preparation and review work should be carefully rigorous.After the specification is completed and preliminary reviewed,metrologists,designers and users on the spot at the same time test one by one according to the calibration specification,complete a full calibration process, and change in time if finding problems.

\section{e) Review of calibration specifications}

The review of calibration specification is an important part of the special test equipment acceptance review meeting.Experts should strictly review the development of contract requirements, specification preparation rules, calibration reports, product test reports and other content.If there is a problem, the relevant personnel will be responsible for the settlement. After the rectification of the problem, submit the review again until the review is passed.

\section{CONCLUSION}

Since it directly affects the performance and technical parameters of military products in debugging, testing, delivery and the following performance in the whole process of the task,special test equipment calibration is one of the key work of military product measurement.The calibration method is correct or not, as is the key to ensure the accuracy of its measurement value.The calibration method is scientific and reasonable or not, as is an important guarantee for accurate and reliable calibration data.Because the nature of each military unit, the 
Informatics Engineering, an International Journal (IEIJ), Vol.4, No.4, December 2016

product and their special test equipment are different.How to carry out the measurement support work of special test equipment for military products, needs each military unit with its own characteristics to explore and practice.

\section{REFERENCES}

[1] Hongzhong Zhu.Measurement \& Management [M].Beijing:Atomic Energy Press,2002.

[2] China Metrology Association. data processing and measurement professional practice [m]. Beijing: China's quality watchdog, 2013.

[3] Yixiong Xu.The Pressure Signal Calibration Technology of the Comprehensive Test System [J].Informatics Engineering,an Internationa Journal,2016,VOL27:01-08.

[4] Sheng Hong Jiang, Feng Yi.Online Calibration of the Sensor [j]. Electronic Design Engineering, 2012,43 (1): 34-37

Authors:

Xu Yi-xiong(1985-), postgraduate in Shanghai University of Engineering Science(SUES), \& Shanghai Institute of Spaceflight Control Technology, the main research interest now is Instrumentation measurement technology, address: Room 1105,Building NO.243,Lane 777,Dushi road, Minhang district, Shanghai(201109), fixed 021-34628108, mobile 13681897190, email: 653341297@qq.com

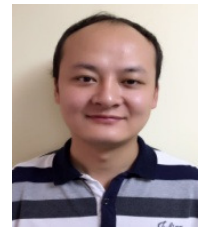

Mu Xiao-nian(1983-),Master of Engineering, Shanghai Institute of Spaceflight Control Technology, the main research interest now is the integrated management.address: Room 201,Building NO.5,Lane 1555,ZhongChun road, Minhang district, Shanghai(201109),email: 819413446@qq.com

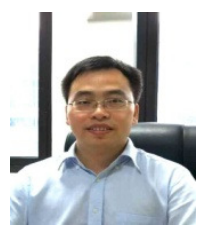

